\title{
Cuidado de enfermería en pacientes con trastorno del espectro autista en un pabellón quirúrgico en clínica privada, Chile
}

\author{
Care of patients with autism spectrum disorder in a surgical ward, Chile
}

\author{
Roberto Lobos ${ }^{1}$, Darynka Parada², Ismael Morales Ojeda ${ }^{3}$, Bárbara Cerda-Aedo ${ }^{4}$
}

\begin{abstract}
RESUMEN
Objetivo: Describir el cuidado de enfermería en pacientes con trastorno del espectro autista dado por los enfermeros que participan de las actividades asistenciales del servicio de pabellón quirúrgico en una clínica privada. Material y métodos: Estudio cuantitativo descriptivo, la muestra correspondió a 40 profesionales de Enfermería que participan en los cuidados de salud dentro del pabellón quirúrgico, quienes atienden a niños y niñas entre 1 a 15 años de edad en una clínica particular de la cuidad de Iquique. Se creó para los enfermeros del servicio, la Encuesta de Conocimiento y Acciones sobre la Atención de Niños y Niñas con TEA (ECA-TEA), la cual permite describir características sociodemográficas, y evaluar los cuidados de enfermería en pabellón hacia niño. Resultados: Se apreció que más del $90 \%$ de los profesionales de esta clínica no tiene capacitación respecto al TEA, lo que disminuye la realización de cuidados adecuados al TEA. Conclusiones: es necesario crear un sistema de apoyo para el profesional de enfermería en la atención de pacientes TEA.
\end{abstract}

Palabras Clave: Trastorno del espectro autista, enfermería, pabellón

\begin{abstract}
Objective: To describe nursing care in patients with autism spectrum disorder given by nurses who participate in the assistance activities of the surgical ward service in a private clinic. Material and methods: Quantitative descriptive study, the sample corresponded to 40 Nursing professionals who participate in health care within the surgical ward, who attend children between 1 and 15 years of age in a private clinic in the city of lquique. For the nurses of the service, the Survey of Knowledge and Actions on the Care of Boys and Girls with ASD (ECA-ASD) was created, which allows describing sociodemographic characteristics, and assessing nursing care in a ward towards children. Results: It was appreciated that more than $90 \%$ of the professionals in this clinic do not have training regarding ASD, which reduces the performance of care appropriate to ASD. Conclusions: It is necessary to create a support system for the nursing professional in the care of ASD patients.
\end{abstract}

Keywords: Autism spectrum disorder, nursing, pavilion

\footnotetext{
${ }^{1}$ EMS Emergencychile, Chillán, Chile.

${ }^{2}$ Universidad Pedro de Valdivia, Valdivia, Chile.

3Universidad Adventista de Chile, Chillán, Chile.

Orcid ID: 0000-0002-1752-7023

${ }^{4}$ Universidad Adventista de Chile, Chillán, Chile.

Orcid ID: 0000-0001-5872-7382
} 


\section{INTRODUCCIÓN}

Atender a un niño con Trastorno del espectro autista (TEA) puede ser complejo y requiere el desarrollo de estrategias que involucran a familia, ya que estos pacientes presentan una comunicación limitada, trastornos de comportamiento, hipersensibilidad a las exploraciones e intolerancia a los cambios en sus rutinas. Además, la atención en salud se dificulta debido a que en muchos casos no podemos conocer con exactitud el tiempo que el niño lleva experimentando molestias, ni la gravedad, intensidad $\mathrm{u}$ origen del malestar (Quijada, 2008).

El estudio "Oportunidades clínicas con niños con signos de autismo", refiere que en los últimos 20 años ha habido un incremento de diagnósticos de este trastorno, estudios realizados por BaronCohen refieren que existe una prevalencia de trastorno del espectro autista en 1 de cada 64 sujetos (citado en Untoiglich, 2013). De igual forma, de un total de 240.569 recién nacidos vivos, se estimó que en el 2009 hubieron 2.156 niños con trastorno del espectro autista (Ministerio de Salud, 2011).

Particularmente en la ciudad de lquique, según datos de las agrupaciones APANDIA (agrupación de padres de niños con disfasia y autismo) y AFANEA (Agrupación de padres y familiares de adolescentes y niños que pertenecen al espectro autista y asperger) mantienen en sus registros en el año 2014, un total de 68 niños.

Como el trastorno del espectro autista (TEA) es una alteración irreversible, no existe recuperación total, sino, solamente estrategias de mejoramiento en su calidad de vida.

A nivel hospitalario, se debe motivar al personal a conocer sobre TEA, de modo que se ejecuten cuidados en base a conocimientos y actualizaciones con pautas básicas en la atención, como: supervisar la presencia de un acompañante significativo en la atención al centro hospitalario, avisar y planificar la hora más adecuada para el ingreso del paciente, permitir la intervención y sugerencias del acompañante en el trato del paciente, realizar valoración de administración de sedación y contención física si el paciente se encuentra agitado, utilizar pictogramas al momento de realizar entrevista con el paciente con trastorno del espectro autista (TEA), supervisar una resolución rápida del equipo de salud en un tiempo mínimo con prioridad de la atención, avisar sobre la patología al decidir traslado al centro hospitalario, ocultar el material médico especialmente llamativo $u$ objeto luminoso al ingreso del servicio, permitir el mantenimiento de objetos personales de uso cotidiano (Parellada, Romo, Boada, Moreno, Llorente, Dorado, s. f.; Quijada, 2008; Taberna, \& Soto, 2013; OMS, 2014).

\section{METODOLOGÍA}

Estudio cuantitativo descriptivo, la muestra correspondió a 40 profesionales de Enfermería que participan en los cuidados de salud dentro del pabellón quirúrgico, quienes atienden a niños y niñas entre 1 a 15 años de edad en una clínica particular de la cuidad de Iquique.

Para este estudio se creó para los enfermeros del servicio, la Encuesta de Conocimiento y Acciones sobre la Atención de Niños y Niñas con TEA (ECA-TEA), la cual permite describir características sociodemográficas, y evaluar los cuidados de enfermería en pabellón hacia niño con TEA. La encuesta fue validada previa a su aplicación con personal de un Centro de Salud Familiar.

Posterior a la aprobación del Comité de Ética de la Clínica, y luego de la aprobación de los responsables de cada área de la clínica se encuestó en los distintos turnos de enfermería. Los datos fueron analizados con Microsoft Excel. Para el análisis estadístico se realizó estadística descriptiva, utilizando en las variables cualitativas frecuencias y porcentajes.

\section{RESULTADOS}

En la tabla 1, se observa que en su mayoría menos de 30 años, con una experiencia menor de cinco. A pesar de los esfuerzos formativos de la profesión por incorporar las situaciones especiales a los cuidados de enfermería, se pudo apreciar que más del $90 \%$ de los profesionales de esta clínica no tiene capacitación respecto al TEA. 
Tabla 1

Datos sociodemográficos de los profesionales de enfermería

\begin{tabular}{|c|c|c|c|}
\hline$n=40$ & & n & $\%$ \\
\hline \multirow[t]{2}{*}{ Sexo } & Masculino & 10 & 25,0 \\
\hline & Femenino & 30 & 75,0 \\
\hline \multirow[t]{3}{*}{ Edad } & 20 a 30 años & 33 & 82,5 \\
\hline & 31 a 40 años & 6 & 15,0 \\
\hline & 41 a 50 años & 1 & 2,5 \\
\hline \multirow[t]{3}{*}{ Experiencia Laboral } & Menos de 5 años & 32 & 80,0 \\
\hline & 6 a 10 años & 7 & 17,5 \\
\hline & 11 a 15 años & 1 & 2,5 \\
\hline \multirow{2}{*}{$\begin{array}{l}\text { Con capacitación respecto a pacientes } \\
\text { con Trastorno del Espectro Autista }\end{array}$} & Si & 1 & 2,5 \\
\hline & No & 39 & 97,5 \\
\hline
\end{tabular}

En la tabla 2, se aprecia como muchos de los cuidados generales que se realizan usualmente con todos los pacientes que van a pabellón, son los incorporados también a los niños con TDA, pero existe aún una dificultad para incluir aquellos cuidados que más tienen relación con el síndrome como lo son: valorar la administración de sedación o contención física, ocultar el material médico llamativo u objeto luminoso, permitir ingresar objetos personales de uso cotidiano, siempre que sean compatibles con las características de la habitación, utilizar pictogramas, entre otros.

Tabla 2

Evaluación de los cuidados de enfermería en pabellón hacia niño con TEA

\begin{tabular}{|c|c|c|c|c|c|c|c|c|c|c|}
\hline \multirow{2}{*}{$n=40$} & \multicolumn{2}{|c|}{ Nunca } & \multicolumn{2}{|c|}{ Rara vez } & \multicolumn{2}{|c|}{$\begin{array}{l}\text { Algunas } \\
\text { veces }\end{array}$} & \multicolumn{2}{|c|}{$\begin{array}{l}\text { Casi } \\
\text { siempre }\end{array}$} & \multicolumn{2}{|c|}{ Siempre } \\
\hline & $\mathrm{n}$ & $\%$ & n & $\%$ & $\mathrm{n}$ & $\%$ & $\mathrm{n}$ & $\%$ & $\mathrm{n}$ & $\%$ \\
\hline $\begin{array}{l}\text { Supervisa la presencia de acompañante significativo en la atención } \\
\text { en el centro hospitalario. }\end{array}$ & 1 & 2,5 & 1 & 2,5 & 4 & 10,0 & 7 & 17,5 & 27 & 67,5 \\
\hline $\begin{array}{l}\text { Permite la intervención y sugerencia del acompañante hasta el } \\
\text { momento de la sedación en pabellón. }\end{array}$ & 2 & 5,0 & 3 & 7,5 & 6 & 15,0 & 21 & 52,5 & 8 & 20,0 \\
\hline $\begin{array}{l}\text { Valora la administración de sedación o contención física si el paciente } \\
\text { se encuentra agitado }\end{array}$ & 8 & 20,0 & 17 & 42,5 & 5 & 12,5 & 3 & 7,5 & 7 & 17,5 \\
\hline $\begin{array}{l}\text { Oculta el material médico llamativo u objeto luminoso al ingreso del } \\
\text { Servicio de Pabellón (sobrecarga sensorial). }\end{array}$ & 24 & 60,0 & 8 & 20,0 & 5 & 12,5 & 2 & 5,0 & 1 & 2,5 \\
\hline Evalúa del dolor utilizando la escala de Wong Baker & 8 & 20,0 & 0 & 0,0 & 0 & 0,0 & 8 & 20,0 & 24 & 60,0 \\
\hline Ubica en un lugar apartado al paciente para disminuir su ansiedad. & 13 & 32,5 & 0 & 0,0 & 13 & 32,5 & 9 & 22,5 & 5 & 12,5 \\
\hline $\begin{array}{l}\text { Avisa o planifica la hora más adecuada para el ingreso del paciente } \\
\text { independiente al cuadro que presente. }\end{array}$ & 12 & 30,8 & 3 & 7,7 & 5 & 12,8 & 8 & 20,5 & 11 & 28,2 \\
\hline $\begin{array}{l}\text { Utiliza pictogramas al momento de realizar la entrevista de ingreso } \\
\text { a Pabellón }\end{array}$ & 13 & 32,5 & 0 & 0,0 & 13 & 32,5 & 9 & 22,5 & 5 & 12,5 \\
\hline $\begin{array}{l}\text { Permite la intervención y sugerencias del acompañante en el trato } \\
\text { de un paciente con Trastorno del Espectro Autista }\end{array}$ & 6 & 15,8 & 3 & 7,9 & 2 & 5,3 & 6 & 15,8 & 21 & 55,3 \\
\hline Solicita la rápida realización de pruebas complementarias & 5 & 13,2 & 4 & 10,5 & 7 & 18,4 & 8 & 21,1 & 14 & 36,8 \\
\hline $\begin{array}{l}\text { Realiza valoración de administración de sedación o contención física } \\
\text { si el paciente se encuentra agitado }\end{array}$ & 7 & 17,5 & 5 & 12,5 & 9 & 22,5 & 3 & 7,5 & 16 & 40,0 \\
\hline $\begin{array}{l}\text { Permite ingresar objetos personales de uso cotidiano, siempre que } \\
\text { sean compatibles con las características de la habitación y con la } \\
\text { funcionalidad de la unidad }\end{array}$ & 8 & 25,8 & 1 & 3,2 & 2 & 6,5 & 7 & 22,6 & 13 & 41,9 \\
\hline $\begin{array}{l}\text { Incluye el calendario de citas programadas para el seguimiento del } \\
\text { problema de salud }\end{array}$ & 4 & 13,3 & 2 & 6,7 & & & 10 & 33,3 & 14 & 46,7 \\
\hline
\end{tabular}




\section{DISCUSIÓN}

En la población estudiada se pudo apreciar que más del $90 \%$ de los profesionales de esta clínica no tiene capacitación respecto al TEA. Lo que es un factor de riesgo considerando las dificultades que se presentan en el tratamiento de esta enfermedad (Pardo-Govea, \& SolísÁñez, 2009). Según la guía de urgencia para el Paciente con Autismo: La sedación y contención física deben ser valoradas siempre en estos pacientes con el fin de evitar la agitación motora, pues la falta o superficialidad en la valoración puede desencadenar situaciones de hostilidad ante la dificultad del niño para comprender su entorno (Parellada et al., s. f.).

Por otro lado, en la tabla 2, se aprecia como muchos de los cuidados generales que se realizan usualmente con todos los pacientes que van a pabellón, son los incorporados también a los niños con TDA, pero existe aún una dificultad para incluir aquellos cuidados que más tienen relación con el síndrome como lo son: valorar la administración de sedación o contención física, ocultar el material médico llamativo u objeto luminoso, permitir ingresar objetos personales de uso cotidiano, siempre que sean compatibles con las características de la habitación, utilizar pictogramas, entre otros (Irarrázaval, Brokering, \& Murillo, 2005).

La aplicación de la escala EVA para el manejo del dolor en un paciente TEA, evidencia falta de conocimiento en los profesionales, pues la forma correcta para medir el dolor en pacientes pediátricos no es con la Escala EVA, sino a través de la Escala Wong Baker donde aparecen imágenes referentes al dolor que el niño siente en ese momento, una herramienta para que el profesional observe gesticulaciones del paciente con TEA sobre todo si no se comunica adecuadamente de manera verbal (Irarrázaval et al., 2005).

Actualmente existe evidencia que apoya la participación de factores genéticos como causa del TEA asociándose a enfermedades monogénicas (como el síndrome de $X$ frágil en donde se puede apreciar una fragilidad en el extremo del cromosoma) manifestando en el niño: comportamiento hiperactivo, déficit atencional, deterioro en el lenguaje (PardoGovea, \& Solís-Áñez, 2009). Esta enfermedad también es de tipo neurobiológica, que se traduce en el daño o alteración ocurrida antes del nacimiento o durante el desarrollo fetal precoz. Estudios en gemelos univitelinos han demostrado que el gemelo de un TEA tiene un $90 \%$ de tener una enfermedad relacionada con este trastorno (Irarrázaval et al., 2005).

Asimismo, existe una tendencia a tener mayor volumen del cerebro en la infancia, debido al aumento de la sustancia blanca. Existiendo pacientes con macrocefalia, aceleración o desaceleración del crecimiento cerebral, disminución del tamaño de las células del sistema límbico y del número de células de Purkinje en el cerebelo (Tellechea, 2013).

En cuanto a la respuesta humoral, se ha encontrado que los niños con TEA presentan niveles elevados de IgE, como evidencia de una respuesta de tipo alérgica. Presentándose hipersensibilidad a grupos específicos de alimentos que contienen caseína y/o gluten, lo que lleva a la postulación de teorías inmunológicas en el trastorno del espectro autista, donde podrían implicarse genes de baja compatibilidad. Asociándose también como causas secundarias al trastorno algunos factores ambientales como: la exposición intrauterina a los virus de la rubéola, citomegalovirus, al ácido valproico y talidomida (Irarrázaval et al., 2005).

Según la guía clínica del Ministerio de Salud (2011) de detección temprana se realiza inicialmente una evaluación del DSPM (Desarrollo Psicomotor) a través de los instrumentos realizados en el control del niño sano como: EEDP (Escala de evaluación de desarrollo psicomotor de 0 a 24 meses) y TEPSI (Test de desarrollo Psicomotor de 24 a 5 años), se evalúa al paciente, y si hubiese algún retraso de lenguaje y desarrollo social es causal para que el profesional de APS (Atención Primaria de Salud) o médico pediatra realice la pauta de cotejo de señales de alerta de TEA, en caso de aparición de una o más señales en su rango etario es motivo de etapa de confirmación diagnóstica. Entre los 16 a 30 meses de edad se deriva a un periodo de vigilancia realizando la aplicación del instrumento M-CHAT/ES (Lista de Modificación para el Autismo) en dos ocasiones, con un intervalo de 30 días entre cada una. Si el resultado es positivo, en ambas aplicaciones, se deriva de forma inmediata a confirmación de diagnóstico. Para efecto de este proceso, deben seguirse los criterios diagnósticos que están establecidos tanto en el manual DSM-IV TR (Diagnóstico y Estadístico de los Trastornos 
Mentales, Texto Revisado), así como en el CIE-10 (Clasificación Internacional de Enfermedades), en los que se describen detalladamente las características clínicas y de comportamiento de cada uno de los trastornos citados (Ministerio de Salud, 2011; Robins, Fein, Barton, \& Green, 2001; Sánchez, \& Blanco, 2008).

Según lo anterior, debe existir en el sistema de salud una preparación al personal en relación al conocimiento sobre la detección precoz ya que esta condición mejorará la calidad de vida del paciente y su entorno. Los estudios sobre la intervención temprana permiten concluir que ésta puede marcar la diferencia entre un pronóstico favorable o desfavorable, ya que diversos trabajos sugieren que, si la intervención se pone en marcha antes de los tres años, se logran efectos mucho más beneficiosos que si se inicia después de los cinco años (Irarrázaval, 2005).

Un estudio realizado en los centros de salud familiar de la ciudad de Catanduva Brasil, refiere que entre más temprano se realiza el diagnóstico, mejores resultados se obtendrán a futuro, por lo tanto, el desconocimiento del síndrome retrasará avance del síndrome y los logros con estos pacientes serán menores (Canal, García, Touriño, Santos, Martín, Ferrari, Martínez, Guisuraga, Boada, Rey, Franco, Fuentes, \& Posada, 2006). Pues para este tratamiento se cuenta con cuatro métodos esenciales para conseguir resultados favorables, entre ellos, se encuentran: Dieta libre de gluten-caseína, Dieta de carbohidratos específicos, Tratamiento para la cándida intestinal (Higuera, 2010).

Entre las funciones del personal de enfermería, es clave e indispensable en las instituciones de salud, lo que le incentiva a mantenerse actualizados en sus conocimientos para la prestación de sus servicios (Cadena, Olvera, Pérez, Balseiro, Miranda, 2012). En este sentido, la Asamblea Mundial de la Salud (OMS, 2014), solicitó con urgencia a los Estados Miembros a incluir las necesidades de las personas afectadas por el trastorno del espectro autista (TEA) y otros desórdenes en el desarrollo, en las políticas y programas vinculados a la salud de los niños y adolescentes, a su desarrollo y salud mental. Esto implica aumentar la capacidad de los sistemas de salud y cuidado social para proveer servicios para pacientes con trastornos del espectro autista y sus familias. También plantea cambiar el enfoque del cuidado en establecimientos de salud pública de atención a largo plazo, hacia servicios no residenciales en la comunidad. Esto significa, además, mejorar la vigilancia en salud para capturar la información sobre los trastornos del espectro autista y asegurarse que los países tengan mejores capacidades para diagnosticar y tratar esta enfermedad (OMS, 2014).

Debido a los resultados de este estudio es que nace la necesidad de crear un sistema de apoyo para el profesional de enfermería en la atención de pacientes TEA, por lo que se ha diseñado un protocolo y flujograma de las acciones en modalidad de sugerencias que debe realizar el personal en los Servicios de Urgencias, Pabellón, Servicio de Hospitalización, Traslado deAmbulancia y Control sano del niño y la niña en Atención Primaria de Salud, y con ello se anexa Check List que servirán a los supervisores de servicios para evaluar el cumplimiento de las acciones con estos pacientes.

\section{Declaración de financiamiento y de conflictos de} interés:

El estudio fue financiado por los autores, quienes declaran no tener algún tipo de conflicto de interés en la investigación realizada.

\section{Correspondencia}

Roberto Lobos

Correo electrónico:

eurlobos@gmail.com

Darynka Parada

Correo electrónico:

darynkap@gmail.com

Ismael Morales Ojeda

Correo electrónico:

ismaelmoralesojeda@gmail.com

Bárbara Cerda-Aedo

Correo electrónico:

psbcerda@gmail.com 


\section{REFERENCIAS BIBLIOGRÁFICAS}

Canal, R., García, P., Touriño, E., Santos, J., Martín, V., Ferrari, M. J., Martínez, M., Guisuraga, Z., Boada, L., Rey, F., Franco, M., Fuentes, J., \& Posada, M. (2006). La detección precoz del autismo. Intervensión Psicosocial, 15(15), 29-47. Recuperado de: http://scielo.isciii.es/pdf/inter/ v15n1/v15n1a03.pdf

Cadena, J., Olvera, S., Pérez, M. T., Balseiro, C., \& Miranda, R. (2016). Evaluación de la capacitación de enfermería en dos institutos nacionales de salud. Enfermería Universitaria, 9(3), 16-24. Recuperado de: http://www.scielo.org.mx/pdf/eu/ v9n3/v9n3a3.pdf

Higuera, M. (2010). Tratamientos biológicos del autismo y dietas de eliminación. Revista Chilena de Pediatría, 81(3), 204-214. Recuperado de: https://scielo.conicyt.cl/pdf/rcp/v81n3/art02.pdf

Irarrázaval O., M. E., Brokering A., W., \& Murillo B., G. A. (2005). Autismo: una mirada desde la psiquiatría de adultos. Revista chilena de neuropsiquiatría, 43(1), 51-60. https://doi.org/10.4067/ S0717-92272005000100007

Ministerio de Salud (2011). Guía de práctica clínica de detección y diagnóstico oportuno de los Trastornos del Espectro Autista (TEA). Santiago, Chile. Recuperado de: http://www.minsal.cl/portal/ url/item/bd81e3a09ab6c3cee040010164012ac2. pdf

OMS (2014). Progresos de la Asamblea Mundial de la Salud en lo concerniente a enfermedades no transmisibles y medicina tradicional [pág. web]. Recuperado de: http://www.who.int/mediacentre/ news/releases/2014/WHA-20140523/es/

Pardo-Govea, T., \& Solís-Áñez, E. (2009). Aspectos inmunogenéticos del autismo. Revisión. Invest Clin Review. Invest Clin, 50(503), 393-406. Recuperado de: http://www.scielo.org.ve/pdf/ic/ v50n3/art13.pdf

Parellada, M., Romo, J., Boada, L., Moreno, C., Llorente, C., \& Dorado, M. (s. f.). Programa de atención médica integral para pacientes con Trastorno del Espectro Autista (AMI-TEA) [documento online]. Recuperado de http:// autismomadrid.es/wp-content/uploads/2012/08/ Memoria-Programa-AMI-TEA-2011.pdf

Quijada, G. C. (2008). Espectro autista. Revista Chilena de Pediatría, 79, 86-91.

Robins, D. L., Fein, D., Barton, M. L., \& Green, J. A. (2001). The Modified Checklist for Autism in Toddlers: an initial study investigating the early detection of autism and pervasive developmental disorders. Journal of Autism and Developmental Disorders, 31(2), 131-44. Recuperado de: http:// www.ncbi.nlm.nih.gov/pubmed/11450812

Sánchez, L., \& Blanco, M. P. (2008). Trastornos del espectro autista, asistencia integral desde Atención Primaria. Pediatría Integral, 12(10), 975-986.

Taberna, S., \& Soto, N. (2013). Agenda de pictogramas para examen de salud Infantil [pág. web]. Recuperado de: http://academica-e. unavarra.es/bitstream/handle/2454/11279/ SoniaTabernalribarren.pdf?sequence $=1$

Tellechea, N. (2013). Trastorno del espectro autista y trastorno específico del lenguaje ¿Dos entidades diferentes o un continuo de manifestaciones neuropsicológicas? Medicina (Buenos Aires) Supl. I(73), 10-15. Recuperado de: http://www. scielo.org.ar/pdf/medba/v73s1/v73s1a03.pdf

Untoiglich, G. (2013). As oportunidades clínicas com crianças com sinais de autismo e seus pais, Estilos Clínicos, 18(3), 543-558. Recuperado de: https://www.revistas.usp.br/estic/article/ viewFile/79866/83823

Recibido: 7 de setiembre Aceptado: 15 de noviembre 